\title{
Predicting hospital stay, mortality and readmission in people admitted for hypoglycaemia: prognostic models derivation and validation
}

\author{
Francesco Zaccardi ${ }^{1}$ - David R. Webb ${ }^{1}$. Melanie J. Davies ${ }^{1} \cdot$ Nafeesa N. Dhalwani $^{1}$. \\ Laura J. Gray ${ }^{2}$ - Sudesna Chatterjee ${ }^{1}$. Gemma Housley ${ }^{3}$ - Dominick Shaw ${ }^{3,4}$. \\ James W. Hatton ${ }^{3} \cdot$ Kamlesh Khunti $^{1}$
}

Received: 30 November 2016 / Accepted: 6 February 2017 / Published online: 17 March 2017

(C) The Author(s) 2017. This article is published with open access at Springerlink.com

\begin{abstract}
Aims/hypothesis Hospital admissions for hypoglycaemia represent a significant burden on individuals with diabetes and have a substantial economic impact on healthcare systems. To date, no prognostic models have been developed to predict outcomes following admission for hypoglycaemia. We aimed to develop and validate prediction models to estimate risk of inpatient death, $24 \mathrm{~h}$ discharge and one month readmission in people admitted to hospital for hypoglycaemia.

Methods We used the Hospital Episode Statistics database, which includes data on all hospital admission to National Health Service hospital trusts in England, to extract admissions for hypoglycaemia between 2010 and 2014. We developed, internally and temporally validated, and compared two prognostic risk models for each outcome. The first model included age, sex, ethnicity, region, social deprivation and Charlson score ('base' model). In the second model, we added to the 'base' model the 20 most common medical conditions and applied a stepwise backward selection of variables ('disease'
\end{abstract}

Electronic supplementary material The online version of this article (doi:10.1007/s00125-017-4235-1) contains peer-reviewed but unedited supplementary material, which is available to authorised users.

Francesco Zaccardi

frazac@fastwebnet.it

1 Diabetes Research Centre, University of Leicester, Leicester General Hospital, Gwendolen Road, Leicester LE5 4PW, UK

2 Department of Health Sciences, University of Leicester, University Road, Leicester, UK

3 Nottingham University Hospitals \& East Midlands Academic Health Science Network, Triumph Road, Nottingham, UK

4 Nottingham Respiratory Research Unit, University of Nottingham, Hucknall Road, Nottingham, UK model). We used C-index and calibration plots to assess model performance and developed a calculator to estimate probabilities of outcomes according to individual characteristics.

Results In derivation samples, 296 out of 11,136 admissions resulted in inpatient death, 1789/33,825 in one month readmission and 8396/33,803 in $24 \mathrm{~h}$ discharge. Corresponding values for validation samples were: 296/10,976, 1207/22,112 and $5363 / 22,107$. The two models had similar discrimination. In derivation samples, $\mathrm{C}$-indices for the base and disease models, respectively, were: $0.77(95 \% \mathrm{CI} 0.75,0.80)$ and $0.78(0.75$, $0.80)$ for death, $0.57(0.56,0.59)$ and $0.57(0.56,0.58)$ for one month readmission, and $0.68(0.67,0.69)$ and $0.69(0.68,0.69)$ for $24 \mathrm{~h}$ discharge. Corresponding values in validation samples were: $0.74(0.71,0.76)$ and $0.74(0.72,0.77), 0.55(0.54,0.57)$ and $0.55(0.53,0.56)$, and $0.66(0.65,0.67)$ and $0.67(0.66,0.68)$. In both derivation and validation samples, calibration plots showed good agreement for the three outcomes. We developed a calculator of probabilities for inpatient death and $24 \mathrm{~h}$ discharge given the low performance of one month readmission models. Conclusions/interpretation This simple and pragmatic tool to predict in-hospital death and $24 \mathrm{~h}$ discharge has the potential to reduce mortality and improve discharge in people admitted for hypoglycaemia.

Keywords Epidemiology $\cdot$ Hypoglycaemia $\cdot$ Inpatient . Mortality $\cdot$ Prognostic model
Abbreviations
HES Hospital Episode Statistics
IMD Index of multiple deprivation
NHS National Health Service 


\section{Introduction}

Hypoglycaemia is the most common side effect of intensive glucose treatment [1]. Severe hypoglycaemic episodes have a negative impact on the quality of life of patients with diabetes and are possibly associated with an increased risk of vascular and nonvascular death [2,3]. Most hypoglycaemic events are mild and self-treated; severe episodes, conversely, require third party support and some result in emergency department and hospital admission, which represents a significant burden on patients and healthcare systems $[1,4]$.

In recent years, several risk prediction models have been proposed for patients admitted to hospital; these models can assist clinicians to define the prognosis and tailor medical decisions [5]. The majority of models have been developed and validated for risk of inpatient death, length of hospital stay and hospital readmission in different clinical settings, mainly in patients with cardiovascular diseases [6-10]. To date, however, no model has been developed for patients admitted to hospital for hypoglycaemia. As these admissions are generally characterised by a lower risk of death $(2-4 \%)$ and a shorter length of stay (usually $<24 \mathrm{~h}$ ) compared with admissions for other medical reasons [6,11-14], the applicability of available prediction models to patients admitted for hypoglycaemia would result in biased risk estimates. Moreover, a risk prediction tool for patients admitted for hypoglycaemia would be particularly useful given the increased trend of patients admitted for hypoglycaemia during the last decade in England and the USA, and the associated use of healthcare resources $[4,11$, $15]$.

In this context, we used Hospital Episode Statistics (HES) admission data from England to develop and validate a risk model for inpatient death, $24 \mathrm{~h}$ discharge and one month readmission for people admitted to hospital for hypoglycaemia.

\section{Methods}

Study design, setting and source of data We extracted data from HES, which contains information on all finished consultant episodes in the National Health Service (NHS) hospital trusts in England (www.hscic.gov.uk/hes, accessed 29 November 2016). All hospital admissions reporting the ICD10 (www.who.int/classifications/icd/en/) diagnosis field E160 (drug-induced hypoglycaemia without coma), E161 (other hypoglycaemia) or E162 (hypoglycaemia, unspecified) in the first position (i.e. hypoglycaemia as the primary reason of admission), and E10+ (insulin-dependent diabetes mellitus) or E11+ (non-insulin-dependent diabetes mellitus) in any of the remaining ICD-10 fields (from second to 20th), were included.

For each admission episode, we collected data on age, sex, self-reported ethnicity, region of usual residence, start and end date of the episode, admission and discharge method (reporting whether admission resulted in death) and Index of Multiple Deprivation (IMD, a weighted index of social deprivation). We used ICD-10 codes to calculate the Charlson comorbidity score [16]. For the current analysis, we defined two temporally distinct derivation and validation samples. In the derivation samples, we included admissions in 2013 for inpatient death and 2010-2012 for one month readmission and length of hospitalisation (defined as $24 \mathrm{~h}$ discharge). Corresponding years for validation samples were 2014 and 2013-2014. We selected these time intervals because trends of hospital admissions for hypoglycaemia were more stable during these years [11].

Models specification We modelled the three outcomes, inpatient death, one month readmission for hypoglycaemia and $24 \mathrm{~h}$ discharge, using complete-case logistic regressions. We developed two prognostic models: the first, defined 'base' model, included age (transformed with a cubic spline with five knots to account for the non-linearity of the relationship between age and hospital admission for hypoglycaemia [11]), sex, ethnicity (white, other), region (East Midlands, London, North East, North West, South East, South West, West Midlands, and Yorkshire and the Humber), social deprivation (deciles of IMD) and Charlson score for all three outcomes. In the second model ('disease'), we added the 20 most common ICD-10 comorbidities reported in positions second to sixth to the base model; comorbidities were identified for each outcome and are reported in ESM Table 1. After their inclusion, we performed a stepwise backward elimination of individual factors using the ordinary Akaike's information criterion to define the final set of variables [17].

Model performance We evaluated the performance of regression models assessing Nagelkerke $R^{2}$, discrimination and calibration. For a specific model, $R^{2}$ indicates the additional variation in the outcomes compared with a model with only the intercept. For a logistic regression, discrimination corresponds to the area under the receiver operating characteristic curve (C-index); a value of 0.5 indicates model discrimination no better than chance, while a value of 1 perfect discrimination [18]. We plotted observed outcomes by decile of predictions to graphically assess calibration, and calculated calibration slope and intercept; values around 1 for slope and 0 for intercept indicate correct calibration [19].

Internal and temporal validation We validated models internally with 300 bootstrap samples to assess possible optimism and temporally by recalculating indices of discrimination, plotting observed vs predicted outcomes, and estimating calibration slope and intercept. Finally, we developed a calculator based on recalibrated models using the calibration slope and intercept obtained in validation samples [20]. 
Guidelines and software for analyses We performed analyses following the general framework proposed by Steyerberg [20] and Harrell [21], and reported results in line with TRIPOD (Transparent Reporting of a multivariable prediction model for Individual Prognosis Or Diagnosis) recommendations (www.tripod-statement.org. accessed 29 November 2016). We used Stata 14.1 and R 3.2.3 (package rms [22]) for all analyses and reported results with $95 \%$ CI. A $p$ value $<0.05$ was considered statistically significant.

\section{Results}

Characteristics of derivation and validation samples Of 22,113 available admissions for inpatient death, one admission $(0.005 \%)$ was excluded due to missing data on age. Of 55,978 available admissions for one month readmission and $24 \mathrm{~h}$ discharge, 41 admissions were excluded for one month readmission $(0.073 \%$; one missing information for age and 40 for social deprivation) and 68 for length of hospital stay ( $0.121 \%$; one missing information for age, 34 for social deprivation, 27 for time to discharge and six for both social deprivation and time to discharge).

Characteristics of the remaining admissions with complete data, by outcome and sample, are reported in Table 1. No major differences were found between the derivation and validation samples. A large proportion of admissions occurred in patients older than 60 years and of white ethnicity. There were slightly more admissions in men than women and Charlson scores were slightly higher in validation samples. The outcome-specific top 20 most common diseases covered approximately $50 \%$ of all reported comorbidities (ESM Table 1). Of these, two for readmission and 15 for $24 \mathrm{~h}$ discharge were included in the final models after the stepwise backward elimination. Associations between variables and outcomes in derivation samples are reported in Fig. 1 for base models and ESM Table 2 for disease models. Performance measures are summarised in Table 2 and calibration plots are depicted in Fig. 2.

Model development and internal validation The base and disease models for inpatient death were developed from 11,136 admissions and 296 (2.7\%) deaths (Table 1). Age and Charlson score were significantly associated with the risk of inpatient death in both the base and disease models (Fig. 1 and ESM Table 2). Discrimination was very similar comparing the two models: the base model showed a C-index of 0.77 (95\% CI $0.75,0.80)$, with minimal over-fitting in bootstrap validation (bias-corrected $\mathrm{C}$-index 0.75 ), while the disease model achieved a $\mathrm{C}$-index of $0.78(0.75,0.80)$ with a biascorrected value of 0.77 (Table 2 ). The prognostic models for one month readmission were derived from 1789 one month readmissions among 33,825 admissions (5.3\%, Table 1).
Ethnicity and region were significantly associated with risk of readmission in both the base and disease model. Discriminations were modest, being C-index $0.57(0.56$, $0.59)$ and $0.57(0.56,0.58)$ for the base and disease models, respectively; bias-corrected $\mathrm{C}$-indices yielded similar results (Table 2). Finally, prognostic models for $24 \mathrm{~h}$ discharge were developed from $839624 \mathrm{~h}$ discharge among 33,803 admissions $(24.8 \%)$. All variables of the base model were associated with $24 \mathrm{~h}$ discharge. In the disease model, 15 further variables were included; of which, 12 were associated with the outcome (ESM Table 2). C-indices were $0.68(0.67,0.69)$ for the base model and $0.69(0.68,0.69)$ for the disease model, with similar bias-corrected values (Table 2). Both models showed good calibration for inpatient death and $24 \mathrm{~h}$ discharge; conversely, one month readmission models had poor calibration, with no spread between deciles of predicted risk (Fig. 2).

Temporal validation The temporal validation of the two models showed values that were slightly lower than those obtained in the derivation sample and very similar when comparing base and disease models (Table 2). For inpatient death (296 events among 10,976 admissions, 2.7\%; Table 1), C-indices were $0.74(0.71,0.76)$ for the base and $0.74(0.72$, 0.77 ) for the disease model (Table 2). Corresponding values for one month readmission (1207 events among 22,112 admissions, 5.5\%; Table 1) were $0.55(0.54,0.57)$ and 0.55 $(0.53,0.56)$, and for $24 \mathrm{~h}$ discharge $(5363$ events among 22,107 admissions, 24.3\%; Table 1), $0.66(0.65,0.67)$ and $0.67(0.66,0.68)$ (Table 2). Calibration plots showed good agreement between observed and predicted risk for inpatient death and $24 \mathrm{~h}$ discharge; however, a slightly higher predicted than observed risk was evident for the base model in the last (tenth) risk group (Fig. 2).

Individual risk calculator Coefficients obtained in the logistic regressions for inpatient death and $24 \mathrm{~h}$ discharge were used to develop an Excel calculator (see ESM) to estimate individual absolute predicted risk based on variables included in the base model (a mobile/desktop app is in production). For both outcomes, models were recalibrated using the calibration slope and intercept estimated in the validation samples (Table 2). We did not include one month readmission because of the poor performance of models for this outcome, and developed the calculator using only base models following criteria of parsimony and simplicity, and given the negligible differences in the performance between the base and disease models. The calculator allows the input of individual data on age, sex, ethnicity, Charlson score and England postcode (for social deprivation) for two patients to visually inspect the impact of changing a single variable. The calculator can be developed in other graphical interfaces by using data reported in Fig. 1 (coefficients), Table 2 (calibration 
Table 1 Characteristics of admissions to hospital for hypoglycaemia

\begin{tabular}{|c|c|c|c|c|c|c|}
\hline \multirow[t]{2}{*}{ Characteristic } & \multicolumn{3}{|l|}{ Derivation } & \multicolumn{3}{|l|}{ Validation } \\
\hline & Inpatient death & One month readmission & $24 \mathrm{~h}$ discharge & Inpatient death & One month readmission & $24 \mathrm{~h}$ discharge \\
\hline Calendar year & 2013 & 2010-2012 & 2010-2012 & 2014 & 2013-2014 & 2013-2014 \\
\hline Admission, $n$ & 11,136 & 33,825 & 33,803 & 10,976 & 22,112 & 22,107 \\
\hline Participant, $n$ & 9937 & 28,554 & 28,533 & 9819 & 19,057 & 19,054 \\
\hline Death, $n$ & 296 & - & - & 296 & - & - \\
\hline Readmission, $n$ & - & 1789 & - & - & 1207 & - \\
\hline $24 \mathrm{~h}$ discharge, $n$ & - & - & 8396 & - & - & 5363 \\
\hline \multicolumn{7}{|l|}{ Age at admission, years } \\
\hline$<20$ & $638(5.7)$ & $2121(6.3)$ & $2121(6.3)$ & $594(5.4)$ & $1232(5.6)$ & $1232(5.6)$ \\
\hline $20-29$ & $334(3.0)$ & $1182(3.5)$ & $1182(3.5)$ & $344(3.1)$ & $678(3.1)$ & $678(3.1)$ \\
\hline $30-39$ & $395(3.6)$ & $1207(3.6)$ & $1206(3.6)$ & $341(3.1)$ & $736(3.3)$ & $736(3.3)$ \\
\hline $40-49$ & $678(6.1)$ & $2008(5.9)$ & 2007 (5.9) & $686(6.3)$ & $1364(6.2)$ & $1362(6.2)$ \\
\hline $50-59$ & $964(8.7)$ & $2730(8.1)$ & $2730(8.1)$ & $960(8.8)$ & $1924(8.7)$ & $1924(8.7)$ \\
\hline $60-69$ & $1542(13.9)$ & $4348(12.9)$ & 4347 (12.9) & $1468(13.4)$ & $3010(13.6)$ & $3010(13.6)$ \\
\hline $70-79$ & $2818(25.3)$ & $9120(27.0)$ & $9118(27.0)$ & $2840(25.9)$ & $5658(25.6)$ & $5656(25.6)$ \\
\hline$\geq 80$ & $3767(33.8)$ & $11,109(32.8)$ & $11,092(32.8)$ & $3743(34.1)$ & $7510(34.0)$ & $7509(34.0)$ \\
\hline \multicolumn{7}{|l|}{ Sex } \\
\hline Women & $5430(48.8)$ & $16,518(48.8)$ & $16,506(48.8)$ & $5257(47.9)$ & $10,687(48.3)$ & $10,686(48.3)$ \\
\hline Men & $5706(51.2)$ & $17,307(51.2)$ & $17,297(51.2)$ & $5719(52.1)$ & $11,425(51.7)$ & $11,421(51.7)$ \\
\hline Charlson index & $2.29 \pm 1.62$ & $2.04 \pm 1.46$ & $2.04 \pm 1.46$ & $2.37 \pm 1.61$ & $2.33 \pm 1.62$ & $2.33 \pm 1.62$ \\
\hline \multicolumn{7}{|l|}{ IMD- $10^{\mathrm{a}}$} \\
\hline Least deprived $10 \%$ & $607(5.5)$ & $1828(5.4)$ & $1826(5.4)$ & $633(5.8)$ & $1240(5.6)$ & $1240(5.6)$ \\
\hline Less deprived 10-20\% & $768(6.9)$ & $2241(6.6)$ & $2240(6.6)$ & $671(6.1)$ & $1439(6.5)$ & $1439(6.5)$ \\
\hline Less deprived $20-30 \%$ & $857(7.7)$ & $2459(7.3)$ & $2458(7.3)$ & $806(7.3)$ & $1663(7.5)$ & $1663(7.5)$ \\
\hline Less deprived $30-40 \%$ & $917(8.2)$ & $2863(8.5)$ & $2861(8.5)$ & $842(7.7)$ & $1759(8.0)$ & $1759(8.0)$ \\
\hline Less deprived $40-50 \%$ & $1027(9.2)$ & $3108(9.2)$ & $3103(9.2)$ & $949(8.7)$ & $1976(8.9)$ & $1976(8.9)$ \\
\hline More deprived $10-20 \%$ & $1436(12.9)$ & $4747(14.0)$ & $4743(14.0)$ & $1563(14.2)$ & 2999 (13.6) & 2999 (13.6) \\
\hline More deprived $20-30 \%$ & $1359(12.2)$ & $4223(12.5)$ & $4222(12.5)$ & $1369(12.5)$ & $2728(12.3)$ & $2727(12.3)$ \\
\hline More deprived $30-40 \%$ & $1195(10.7)$ & $3538(10.5)$ & $3537(10.5)$ & $1192(10.9)$ & $2387(10.8)$ & $2387(10.8)$ \\
\hline More deprived $40-50 \%$ & $1101(9.9)$ & $3314(9.8)$ & $3312(9.8)$ & $1174(10.7)$ & $2275(10.3)$ & $2273(10.3)$ \\
\hline Most deprived $10 \%$ & $1869(16.8)$ & $5504(16.3)$ & $5501(16.3)$ & $1777(16.2)$ & $3646(16.5)$ & $3644(16.5)$ \\
\hline \multicolumn{7}{|l|}{ Ethnicity } \\
\hline White & $9225(82.8)$ & $28,185(83.3)$ & $28,166(83.3)$ & $9030(82.3)$ & $18,255(82.6)$ & $18,250(82.6)$ \\
\hline Other & $1911(17.2)$ & $5640(16.7)$ & $5637(16.7)$ & $1946(17.7)$ & $3857(17.4)$ & $3857(17.4)$ \\
\hline
\end{tabular}

Data reported as number (percentage) or mean $\pm \mathrm{SD}$

${ }^{\mathrm{a}}$ IMD score in deciles

Complete-case data (i.e. non-missing) are shown - there was one missing information for inpatient death (age), 41 for readmission (one age and 40 IMD10 ) and 68 for length of hospital stay (one age, 34 IMD-10, 27 time to discharge and six both IMD-10 and time to discharge)

slope and intercept) and ESM Excel file (spline transformation of age).

\section{Discussion}

Main findings Using a large sample of hospital admissions for hypoglycaemia in England, we developed, internally and temporally validated and calibrated two prognostic models for length of hospital stay, inpatient death and readmission. The two models performed well in terms of fitting $\left(R^{2}\right.$ similar to risk models in other clinical setting [23, 24]) and calibration, and did not meaningfully differ in the prediction of inpatient death and length of hospital stay, defined in this study as same-day discharge. Conversely, models failed to accurately predict one month readmission for hypoglycaemia. In fact, the same variables used for inpatient death and length of stay did not accurately predict the risk for one month readmissions, 


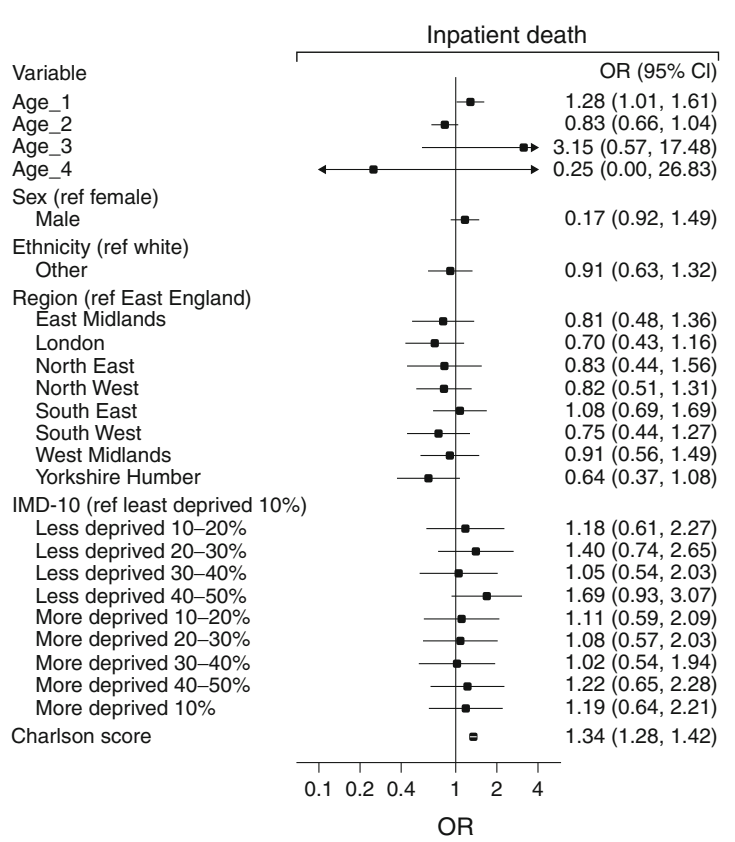

Fig. 1 Associations of variables with outcomes for base model (derivation samples). Age 1, Age 2, Age 3 and Age 4 indicate restricted cubic spline transformation of age. ORs are reported per unit increase of

underlying the possibility that other, unmeasured factors are more relevant in identifying patients at higher risk of recurrent admissions for hypoglycaemia. For all outcomes, model performances were similar in temporal validations. These analyses allowed the development of a tool to assess individual risk based on basic information that are routinely collected in patients admitted to NHS hospital trusts in England.

Interpretation in the context of available evidence In the last decade, hospital admissions for hypoglycaemia have consistently increased in England, as well as in the USA [11, 15]. Although only a fraction of hypoglycaemic episodes result in hospitalisation, admissions are generally reserved for patients potentially at higher risk of complications and have
One month readmission

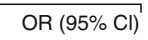

$1.00(0.99,1.01)$ $0.98(0.97,1.00)$ $1.45(1.09,1.94)$ $0.19(0.04,0.82)$

$0.02(0.93,1.12)$ $0.68(0.58,0.79)$ $0.84(0.66,1.07)$ $1.34(1.09,1.64)$ $0.97(0.76,1.25)$ $1.14(0.93,1.38)$ $0.84(0.68,1.05)$ $0.80(0.63,1.03)$ $0.90(0.72,1.12)$
$1.10(0.89,1.37)$ $0.83(0.62,1.12)$ $0.80(0.59,1.07)$ $0.98(0.74,1.28)$ $1.02(0.78,1.34)$ $1.17(0.91,1.50)$ $1.07(0.83,1.38)$ $1.04(0.80,1.35)$ $1.02(0.78,1.33)$ $1.07(0.83,1.37)$ $1.03(1.00,1.07)$

$\begin{array}{lllllll} & 1 & 0.2 & 0.4 & 0.7 & 1 & 1.52\end{array}$

OR
$24 \mathrm{~h}$ discharge

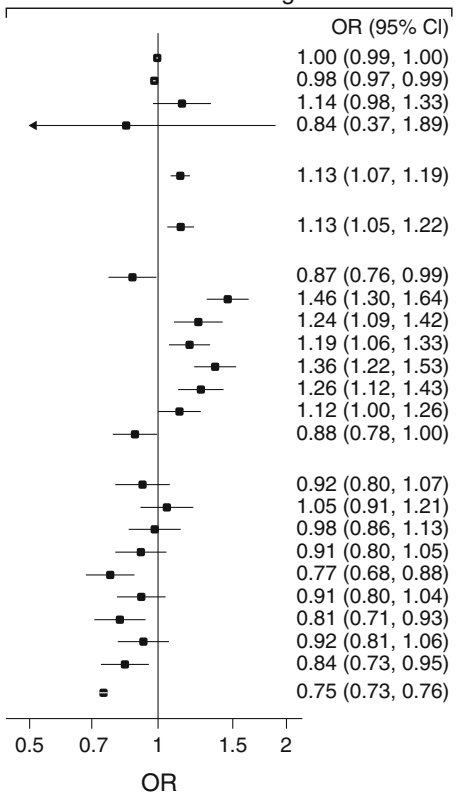

Charlson score. Constants of the models were: -16.864 for inpatient mortality, -2.701 for one month readmission and 0.086 for $24 \mathrm{~h}$ discharge

considerable resource implications for national healthcare systems [4]. Studies have also shown that a significant proportion of admissions for hypoglycaemia occur in people previously admitted for the same reason $[11,15]$. Therefore, the availability of prognostic models for length of hospital stay, risk of death and hospital readmission may be a useful tool to support clinicians and decision makers.

In recent years, various clinical risk models for inpatient death have been developed and validated in different clinical settings, including people with myocardial infarction [6], valve replacement [7] or abdominal aortic aneurism [8], or those admitted to intensive care units [25]. Similarly, validated models for readmissions are available for all-cause and causespecific readmissions, such as cardiovascular, gastrointestinal

Table 2 Model performance indices in derivation and validation samples

\begin{tabular}{|c|c|c|c|c|c|c|c|}
\hline \multirow[t]{2}{*}{ Model } & \multicolumn{3}{|l|}{ Derivation } & \multicolumn{4}{|l|}{ Validation } \\
\hline & $\begin{array}{l}\text { Nagelkerke } \\
R^{2}(\%)\end{array}$ & $\begin{array}{l}\text { C-index } \\
(95 \% \mathrm{CI})\end{array}$ & $\begin{array}{l}\text { Bias-corrected } \\
\text { C-index }\end{array}$ & $\begin{array}{l}\text { Nagelkerke } \\
R^{2}(\%)\end{array}$ & $\begin{array}{l}\text { C-index } \\
(95 \% \mathrm{CI})\end{array}$ & $\begin{array}{l}\text { Calibration } \\
\text { slope }\end{array}$ & $\begin{array}{l}\text { Calibration } \\
\text { intercept }\end{array}$ \\
\hline \multicolumn{8}{|c|}{ Inpatient death } \\
\hline Base & 12.1 & $0.77(0.75,0.80)$ & 0.75 & 8.1 & $0.74(0.71,0.76)$ & 0.77 & 0.00 \\
\hline Disease & 11.8 & $0.78(0.75,0.80)$ & 0.77 & 9.0 & $0.74(0.72,0.77)$ & 0.86 & -0.01 \\
\hline \multicolumn{8}{|c|}{ One month readmission } \\
\hline Base & 1.0 & $0.57(0.56,0.59)$ & 0.56 & 0.5 & $0.55(0.54,0.57)$ & 0.70 & 0.03 \\
\hline Disease & 0.9 & $0.57(0.56,0.58)$ & 0.56 & 0.4 & $0.55(0.53,0.56)$ & 0.66 & 0.03 \\
\hline \multicolumn{8}{|c|}{$24 \mathrm{~h}$ discharge } \\
\hline Base & 10.6 & $0.68(0.67,0.69)$ & 0.68 & 8.4 & $0.66(0.65,0.67)$ & 0.83 & 0.04 \\
\hline Disease & 11.1 & $0.69(0.68,0.69)$ & 0.69 & 8.9 & $0.67(0.66,0.68)$ & 0.82 & 0.05 \\
\hline
\end{tabular}



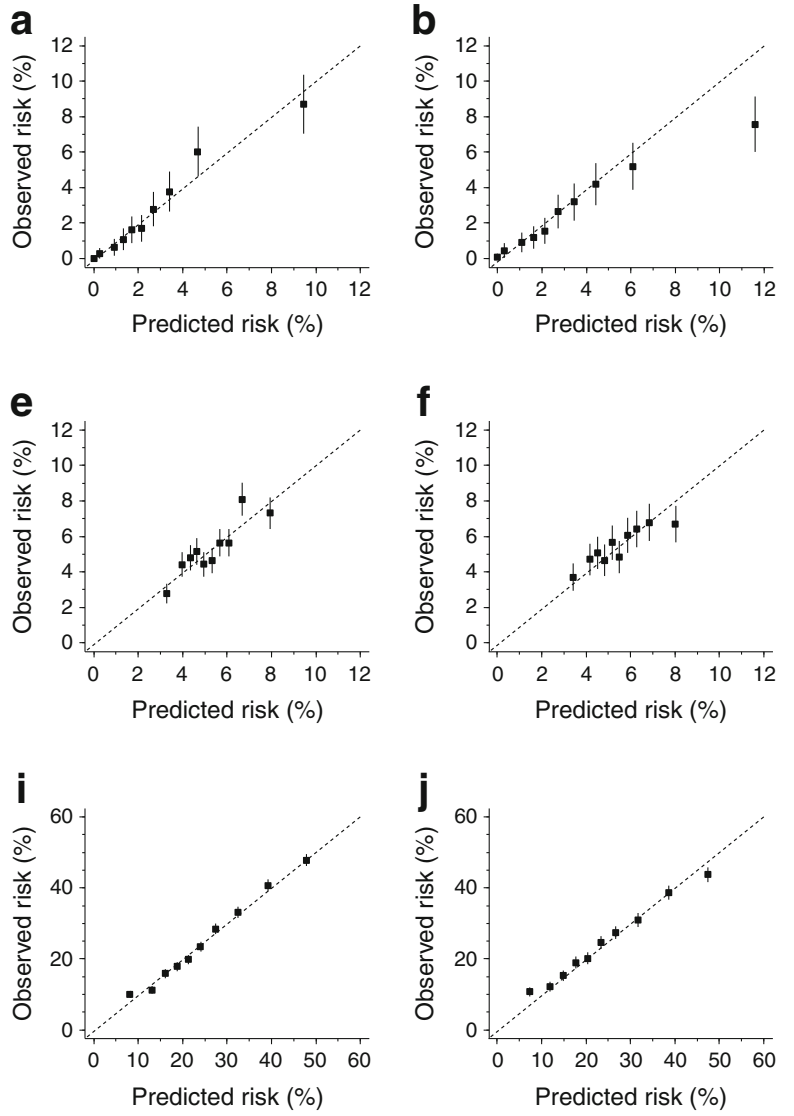

Fig. 2 Calibration plots for base and disease models in derivation and validation samples. Inpatient death: base model, derivation (a) and validation (b) sample; disease model, derivation (c) and validation (d) sample. One month readmission: base model, derivation (e) and validation (f)

or pulmonary diseases [9, 26-30]. More limited, on the other hand, are validated models for length of hospital stay for patients with, for example, chronic obstructive pulmonary disease [31], gastrointestinal bleeding [32] or stroke [10]. Studies aiming to develop clinical prediction models are appreciably different in terms of variable accessibility, model specification procedures, temporal and geographical settings and, most importantly, population studied. It is therefore not surprising that the final variables included in the models, the strength of their associations with outcomes and the occurrence of the outcomes are inconsistent across studies. This is in part due to differences in the aetiology and pathophysiology of diseases (which could influence, for example, the selection of variables) as well as to differences in their severity (for example, risk of inpatient death following decompensated heart failure vs hypoglycaemia). Therefore, the precise definition of a homogeneous population to whom the prediction models apply is of crucial importance.

Strength and limitations To our knowledge, no model has been developed to date to predict hospital outcomes in patients admitted for hypoglycaemia. A major strength of this study is
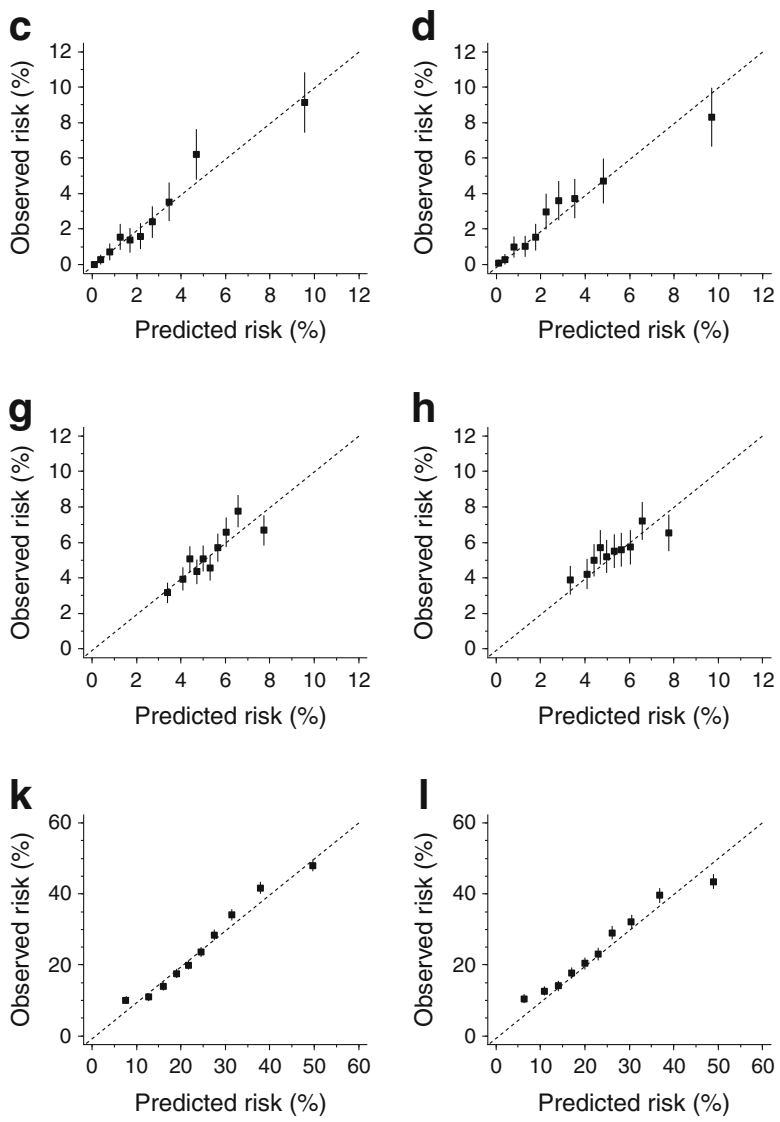

sample; disease model, derivation (g) and validation (h) sample. $24 \mathrm{~h}$ discharge: base model, derivation (i) and validation (j) sample; disease model, derivation (k) and validation (l) sample. Error bars indicate 95\% CI

the availability of a large nationwide database with detailed information on hospital admissions. Furthermore, information was missing only in a very low proportion of admissions, and models were internally and temporally validated.

At the same time, several points should be considered for the interpretation of these findings. First, in all models we used only variables available in the HES database. We may not have included important prognostic variables that could be particularly relevant for one month readmission. Insulin therapy and diabetes duration are related to a higher risk of severe hypoglycaemia and might confound the association between factors included in the analysis, such as age and comorbidities, and risk of readmission [33]. Lack of detailed data on glucoselowering therapies could explain the low performance of models for this outcome. Similarly, lack of information on attendance at educational programmes to avoid recurrent severe hypoglycaemia might have influenced our results. It should be noted, however, that a recent systematic review has confirmed initial observations about the poor to moderate performance of risk models for one month hospital readmissions [28], even in those including an extensive panel of potential predictors [9]. Moreover, although several studies 
have evidenced multiple clinical risk factors for severe hypoglycaemia, the large majority of these analyses reported only associations that do not necessarily translate into better prognostic ability [34].

Second, the ICD-10 codes E10+ (insulin-dependent diabetes mellitus) and E11+ (non-insulin-dependent diabetes mellitus) have been used only to define the population under investigation (people with diabetes) and could not be considered as a proxy of treatment. Indeed, it is possible that individuals with insulintreated type 2 diabetes have been coded as E11+. Conversely, while in principle E10+ and E11+ should respectively identify individuals with type 1 and type 2 diabetes, we could not exclude that some E10+ patients had insulin-treated type 2 diabetes. Finally, for some patients the HES data has inconsistent coding of E10+/E11+ over time (change of diabetes type). Therefore, we could not clearly separate the two groups for the analyses as they included non-well-phenotyped patients.

Third, variable selection in prognostic models is well recognised as the most difficult step in model development. At two extremes, selection of variables can be based only on the expert knowledge of subject matter or only on statistical methods, although the latter approach has been criticised for unstable selection of predictors and bias estimation of associations [20]. In this analysis, we developed a simple model, responding to criteria of parsimony and clinical knowledge, based on six simple items of information, and a second model, with more detailed specification of comorbidities, based on a statistical method to define the final set of variables. The performance of the two models, however, was very similar and justified the use of the variables in the base model for predicting individual risk.

Fourth, HES data are routinely collected for administrative rather than research purposes; as such, there is some potential for inaccuracies in data collection and recording.

Finally, we only considered death occurring during hospitalisation and not short- or long-term mortality after discharge, and investigated readmissions only for hypoglycaemia.

Clinical and research implications These prediction models were developed to estimate individual-level risk using simple clinical and demographic data. The models for length of stay and inpatient death performed well and, along with clinical judgement, could be used by decision makers to personalise targets and strategies. On the other hand, models failed to predict hospital readmission accurately. Given the substantial cost associated with hospital readmissions in the UK and the high prevalence of one month readmission in patients admitted for hypoglycaemia $[11,15,35]$, further studies are warranted to address this important knowledge, clinical and public health gap.

Notwithstanding the importance and the implications of length of stay as a quality indicator across hospitals [36], there are still methodological uncertainties about the best modelling approach to analyse such data and further research is required. Logistic regression estimating discharge at meaningful time points, time-to-event analysis or mixture models have been suggested, with unclear advantages in simulations studies of one method over another [37-39]. Accounting for HES database characteristics, we opted to perform a logistic regression using $24 \mathrm{~h}$ as the specific time point. Indeed, in HES length of stay can be calculated as the difference between two dates, thus resulting in admissions of length of zero ( $24 \mathrm{~h}$ discharge, i.e. same date for entry and exit) or multiples of 1 day. As about $25 \%$ of discharges occurred within $24 \mathrm{~h}$, time-to-event analysis was not a suitable approach to analyse these data. In similar circumstances where a significant proportion of discharges occur within $24 \mathrm{~h}$, a more detailed description of length of hospitalisation with time-to-event analysis is possible only if length of stay is reported in fraction of day (i.e. hours).

We performed a validation of the models for admissions during two different periods (temporal validation). These results, therefore, pertain only to admissions for hypoglycaemia in England and full external validation (temporal and spatial) is required to validate models accounting for geographical and temporal differences.

Conclusion A prediction model for risk of inpatient death and $24 \mathrm{~h}$ discharge in individuals admitted to hospital for hypoglycaemia has been developed and validated. Individual risk can be estimated using simple information that is collected routinely in hospitalised patients. While further studies are required to validate this model and to assess the relevance of glucose-lowering therapies in risk prediction, this simple and pragmatic tool can improve the quality of care through personalised approaches and optimise resource allocation.

\section{Note added in proof}

During the review process of the manuscript, this research has been accepted as:

1. Poster at the Diabetes UK Professional Conference (8-10 March 2017, Manchester, UK)

2. Oral presentation at the Informatics for Health Congress (24-26 April 2017, Manchester, UK)

Acknowledgements We acknowledge support from the National Institute for Health Research Collaboration for Leadership in Applied Health Research and Care - East Midlands (NIHR CLAHRC - EM), the Leicester Clinical Trials Unit and the NIHR LeicesterLoughborough Diet, Lifestyle and Physical Activity Biomedical Research Unit, which is a partnership between University Hospitals of Leicester NHS Trust, Loughborough University and the University of Leicester. The views expressed are those of the authors and not necessarily those of the NHS, the NIHR or the Department of Health. HES were made available by the NHS Health and Social Care Information Centre (copyright 2012, reused with the permission of the Health and Social Care Information Centre). All rights reserved. 
Data availability $\mathrm{FZ}$ and GH had full access to the dataset under the terms of the Hospital Episode Statistics Data Re-Use Agreement. The raw data extract is subject to a data sharing agreement between the authors and Hospital Episode Statistics Data Re-Use terms. Under these terms data may not be made publicly available. Statistical codes are available from the corresponding author (FZ).

Funding source FZ is a Clinical Research Fellow funded with an unrestricted educational grant from Sanofi-Aventis to the University of Leicester. The funding source had no role in study design, data collection, data analysis, data interpretation or writing of the report.

Duality of interest $\mathrm{KK}$ has acted as a consultant and speaker for Novartis, Novo Nordisk, Sanofi-Aventis, Lilly and Merck Sharp \& Dohme. He has received grants in support of investigator and investigator initiated trials from Novartis, Novo Nordisk, Sanofi-Aventis, Lilly, Pfizer, Boehringer Ingelheim and Merck Sharp \& Dohme. KK has received funds for research, honoraria for speaking at meetings and has served on advisory boards for Lilly, Sanofi-Aventis, Merck Sharp \& Dohme and Novo Nordisk.

MJD has acted as consultant, advisory board member and speaker for Novo Nordisk, Sanofi-Aventis, Lilly, Merck Sharp \& Dohme, Boehringer Ingelheim, AstraZeneca and Janssen, and as a speaker for Mitsubishi Tanabe Pharma Corporation. She has received grants in support of investigator and investigator initiated trials from Novo Nordisk, Sanofi-Aventis and Lilly.

DRW has received grants in support of investigator initiated studies and honoraria from Sanofi-Aventis and Novo Nordisk.

$\mathrm{SC}$ has received speaker fees and/or educational funding from Janssen, Eli Lilly, Novo Nordisk, Astra Zeneca and Boehringer Ingelheim, and grants in support of investigator initiated trials from Boehringer Ingelheim and Janssen.

All other authors declare no duality of interest in relation to their involvement in this manuscript.

Author contribution All authors provided substantial contributions to study conception, design and interpretation of data. FZ performed all statistical analyses. GH extracted HES data. All authors were involved in drafting the article or revising it critically for important intellectual content, and approved the final version to be published. Neither this manuscript nor one with substantially similar content has been published or is being considered for publication elsewhere. FZ is the study guarantor.

Open Access This article is distributed under the terms of the Creative Commons Attribution 4.0 International License (http:// creativecommons.org/licenses/by/4.0/), which permits unrestricted use, distribution, and reproduction in any medium, provided you give appropriate credit to the original author(s) and the source, provide a link to the Creative Commons license, and indicate if changes were made.

\section{References}

1. Cryer PE (2008) The barrier of hypoglycemia in diabetes. Diabetes 57:3169-3176

2. Barendse S, Singh H, Frier BM, Speight J (2012) The impact of hypoglycaemia on quality of life and related patient-reported outcomes in type 2 diabetes: a narrative review. Diabet Med 29:293302

3. Khunti K, Davies M, Majeed A, Thorsted BL, Wolden ML, Paul SK (2015) Hypoglycemia and risk of cardiovascular disease and all-cause mortality in insulin-treated people with type 1 and type 2 diabetes: a cohort study. Diabetes Care 38:316-322
4. McEwan P, Larsen Thorsted B, Wolden M, Jacobsen J, Evans M (2015) Healthcare resource implications of hypoglycemia-related hospital admissions and inpatient hypoglycemia: retrospective record-linked cohort studies in England. BMJ Open Diabetes Res Care 3:e000057

5. Steyerberg EW, Moons KG, van der Windt DA et al (2013) Prognosis Research Strategy (PROGRESS) 3: prognostic model research. PLoS Med 10:e1001381

6. McNamara RL, Kennedy KF, Cohen DJ et al (2016) Predicting in-hospital mortality in patients with acute myocardial infarction. J Am Coll Cardiol 68:626-635

7. Edwards FH, Cohen DJ, O'Brien SM et al (2016) Development and validation of a risk prediction model for in-hospital mortality after transcatheter aortic valve replacement. JAMA Cardiology 1:46-52

8. van Beek SC, Blankensteijn JD, Balm R, Dutch Randomised Endovascular Aneurysm Management trial collaborators (2013) Validation of three models predicting in-hospital death in patients with an abdominal aortic aneurysm eligible for both endovascular and open repair. J Vasc Surg 58:1452-1457

9. Zhou H, Della PR, Roberts P, Goh L, Dhaliwal SS (2016) Utility of models to predict 28-day or 30-day unplanned hospital readmissions: an updated systematic review. BMJ Open 6:e011060

10. Kwok CS, Clark AB, Musgrave SD et al (2015) The SOAR stroke score predicts hospital length of stay in acute stroke: an external validation study. Int J Clin Pract 69:659-665

11. Zaccardi F, Davies MJ, Dhalwani NN et al (2016) Trends in hospital admissions for hypoglycaemia in England: a retrospective, observational study. Lancet Diabetes Endocrinol 4:677-685

12. Harries TH, Thornton HV, Crichton S, Schofield P, Gilkes A, White PT (2015) Length of stay of COPD hospital admissions between 2006 and 2010: a retrospective longitudinal study. Int J Chron Obstruct Pulmon Dis 10:603-611

13. Loudon BL, Gollop ND, Carter PR, Uppal H, Chandran S, Potluri $\mathrm{R}$ (2016) Impact of cardiovascular risk factors and disease on length of stay and mortality in patients with acute coronary syndromes. Int J Cardiol 220:745-749

14. Kajimoto K, Sato N, Keida T et al (2013) Association between length of stay, frequency of in-hospital death, and causes of death in Japanese patients with acute heart failure syndromes. Int J Cardiol 168:554-556

15. Lipska KJ, Ross JS, Wang Y et al (2014) National trends in US hospital admissions for hyperglycemia and hypoglycemia among Medicare beneficiaries, 1999 to 2011. JAMA Intern Med 174: 1116-1124

16. Charlson ME, Pompei P, Ales KL, MacKenzie CR (1987) A new method of classifying prognostic comorbidity in longitudinal studies: development and validation. J Chronic Dis 40:373-383

17. Lawless JF, Singhal K (1978) Efficient screening of non-normal regression-models. Biometrics 34:318-327

18. Harrell FE Jr, Califf RM, Pryor DB, Lee KL, Rosati RA (1982) Evaluating the yield of medical tests. JAMA 247:2543-2546

19. Steyerberg EW, Vickers AJ, Cook NR et al (2010) Assessing the performance of prediction models: a framework for traditional and novel measures. Epidemiology 21:128-138

20. Steyerberg EW (2009) Clinical prediction models: a practical approach to development, validation, and updating. Springer, New York

21. Harrell FE Jr (2015) Regression modeling strategies with applications to linear models, logistic and ordinal regression, and survival analysis, 2nd edn. Springer, New York

22. Harrell FE Jr. (2016) Regression modeling strategies. R package version 4.5-0. Available from https://CRAN.R-project.org/ package $=$ rms, accessed 29 November 2016

23. Battes L, Barendse R, Steyerberg EW et al (2013) Development and validation of a cardiovascular risk assessment model in patients with established coronary artery disease. Am J Cardiol 112:27-33 
24. Traeger AC, Henschke N, Hubscher M et al (2016) Estimating the risk of chronic pain: development and validation of a prognostic model (PICKUP) for Patients with acute low back pain. PLoS Med 13:e1002019

25. Paul E, Bailey M, Pilcher D (2013) Risk prediction of hospital mortality for adult patients admitted to Australian and New Zealand intensive care units: development and validation of the Australian and New Zealand Risk of Death model. J Crit Care 28:935-941

26. Donze J, Aujesky D, Williams D, Schnipper JL (2013) Potentially avoidable 30-day hospital readmissions in medical patients: derivation and validation of a prediction model. JAMA Intern Med 173: 632-638

27. Donze JD, Williams MV, Robinson EJ et al (2016) International validity of the HOSPITAL score to predict 30-day potentially avoidable hospital readmissions. JAMA Intern Med 176:496-502

28. Kansagara D, Englander H, Salanitro A et al (2011) Risk prediction models for hospital readmission: a systematic review. JAMA 306: $1688-1698$

29. Robusto F, Lepore V, D'Ettorre A et al (2016) The Drug Derived Complexity Index (DDCI) Predicts mortality, unplanned hospitalization and hospital readmissions at the population level. PLoS One 11:e0149203

30. van Walraven C, Dhalla IA, Bell C et al (2010) Derivation and validation of an index to predict early death or unplanned readmission after discharge from hospital to the community. CMAJ 182: 551-557

31. Quintana JM, Unzurrunzaga A, Garcia-Gutierrez S et al (2015) Predictors of hospital length of stay in patients with exacerbations of COPD: a cohort study. J Gen Intern Med 30:824-831
32. Saltzman JR, Tabak YP, Hyett BH, Sun X, Travis AC, Johannes RS (2011) A simple risk score accurately predicts in-hospital mortality, length of stay, and cost in acute upper GI bleeding. Gastrointest Endosc 74:1215-1224

33. Budnitz DS, Lovegrove MC, Shehab N, Richards CL (2011) Emergency hospitalizations for adverse drug events in older Americans. N Engl J Med 365:2002-2012

34. Lo A, Chernoff H, Zheng T, Lo SH (2015) Why significant variables aren't automatically good predictors. Proc Natl Acad Sci U S A $112: 13,892-13,897$

35. Department of Health. 2012. Progress in making NHS efficiency savings. National Audit Office, London. Available from www.nao. org.uk/wp-content/uploads/2012/12/1213686.pdf, accessed 29 November 2016

36. NHS Institute for Innovation. Quality and service improvement tools. Available from www.institute.nhs.uk/quality and service improvement tools/quality and service improvement tools/ length_of_stay.html, accessed 29 November 2016

37. Verburg IW, de Keizer NF, de Jonge E, Peek N (2014) Comparison of regression methods for modeling intensive care length of stay. PLoS One 9:e109684

38. Brock GN, Barnes C, Ramirez JA, Myers J (2011) How to handle mortality when investigating length of hospital stay and time to clinical stability. BMC Med Res Methodol 11:144

39. Atienza N, Garcia-Heras J, Munoz-Pichardo JM, Villa R (2008) An application of mixture distributions in modelization of length of hospital stay. Stat Med 27:1403-1420 